\title{
SN 2007ax: AN EXTREMELY FAINT TYPE Ia SUPERNOVA
}

\author{
M. M. Kasliwal ${ }^{1,2}$ E. O. Ofek, ${ }^{1}$ A. Gal-Yam, ${ }^{1}$ A. Rau, ${ }^{1}$ P. J. Brown, ${ }^{3}$ S. B. Cenko, ${ }^{4}$ P. B. Cameron, ${ }^{1}$ R. Quimby, ${ }^{1}$ \\ S. R. Kulkarni, ${ }^{1}$ L. Bildsten, ${ }^{5}$ P. Milne, ${ }^{6}$ And G. Bryngelson ${ }^{7}$ \\ Received 2008 January 16; accepted 2008 July 3; published 2008 July 23
}

\begin{abstract}
We present multiband photometric and optical spectroscopic observations of SN 2007ax, the faintest and reddest Type Ia supernova ( $\mathrm{SN}$ Ia) yet observed. With $M_{B}=-15.9$ and $(B-V)_{\max }=1.2$, this $\mathrm{SN}$ is over half a magnitude fainter at maximum light than any other SN Ia. Similar to subluminous SN 2005ke, SN 2007ax also appears to show excess in UV emission at late time. Traditionally, $\Delta m_{15}(B)$ has been used to parameterize the decline rate for SNe Ia. However, the $B$-band transition from fast to slow decline occurs sooner than 15 days for faint SNe Ia. Therefore we suggest that a more physically motivated parameter, the time of intersection of the two slopes, be used instead. Only by explaining the faintest (and the brightest) supernovae can we thoroughly understand the physics of thermonuclear explosions. We suggest that future surveys should carefully design their cadence, depth, pointings, and follow-up to find an unbiased sample of extremely faint members of this subclass of faint SNe Ia.
\end{abstract}

Subject headings: supernovae: individual (SN 2007ax, SN 1991bg, SN 1999by, SN 2005ke) supernovae: general - ultraviolet: stars

Online material: color figure

\section{INTRODUCTION}

Inspired by the application as a standard cosmological candle, the progress in understanding Type Ia supernovae ( $\mathrm{SNe}$ Ia) has grown in leaps and bounds. However, the understanding of their weakest subluminous cousins has been purposefully overlooked as their atypical light curve and atypical spectra make them contaminants for cosmological studies. We suggest here some characteristics that make the physics of the explosions of faint $\mathrm{SNe}$ Ia intriguing in their own right.

In this Letter, we present SN 2007ax which, with a peak absolute magnitude of $M_{B}=-15.9$ and $(B-V)_{\max }=1.2$, is the faintest and reddest Type Ia supernova yet discovered. Although the class of SNe Ia is remarkably homogenous, subluminous SNe Ia show atypical spectral and light curve features (Garnavich et al. 2004; Taubenberger et al. 2008). Photometrically, not only do they fade much faster than predicted by the Phillips relation, they are also very red at maximum and (at least SN 2005ke and SN 2007ax) appear to show UV excess at late time. Spectroscopically, they have broad Ti II features and moderate expansion velocities.

SN 2007ax was discovered in NGC 2577, at $\alpha=$ $08^{\mathrm{h}} 22^{\mathrm{m}} 43.23^{\mathrm{s}}, \delta=22^{\circ} 33^{\prime} 16.9^{\prime \prime}$, on UT 2007 March 21.978 by Arbour (2007) at an unfiltered magnitude of 17.2. Upper limits of >18.5 mag on March 17.636 and >19.0 mag on March 9.959 were also reported. Spectra obtained on March 26 by Blondin et al. (2007) and Morrell \& Folatelli (2007) showed that it was a SN Ia near maximum light similar to SN 1991bg.

\footnotetext{
${ }^{1}$ Astronomy Department, California Institute of Technology, 105-24, Pasadena, CA 91125; mansi@astro.caltech.edu.

${ }^{2}$ George Ellory Hale Fellow, Gordon and Betty Moore Foundation.

${ }^{3}$ Department of Astronomy and Astrophysics, Pennsylvania State University, 525 Davey Laboratory, University Park, PA 16802.

${ }^{4}$ Space Radiation Laboratory, California Institute of Technology, MS 22047, Pasadena, CA 91125.

${ }^{5}$ Kavli Institute for Theoretical Physics and Department of Physics, Kohn Hall, University of California, Santa Barbara, CA 93106.

${ }^{6}$ Steward Observatory, 933 North Cherry Avenue, Tucson, AZ 85721.

${ }^{7}$ Department of Physics and Astronomy, Clemson University, 118 Kinard Laboratory, Clemson, SC 29634.
}

In this Letter, we present multiepoch, multiband imaging and spectroscopic follow-up of SN 2007ax including optical, ultraviolet, and near-infrared. We summarize our observations in $\S 2$, present our analysis and comparison with other faint $\mathrm{SNe}$ Ia in $\S 3$, and discuss possible scenarios for faint thermonuclear explosions in $\S 4$. We conclude with how future surveys can systematically design their cadence, limiting magnitude, and pointings to search for more members belonging to this subclass of faint SNe Ia.

\section{OBSERVATIONS AND DATA REDUCTION}

The automated Palomar 60 inch $(1.5 \mathrm{~m})$ telescope (Cenko et al. 2006) started daily observations of SN 2007ax on UT 2007 March 29 in $g^{\prime}$ and $r^{\prime}$ bands. Data were reduced using custom routines. Aperture photometry was done after image subtraction using two custom modifications of the ISIS algorithm (Alard \& Lupton 1998), hotpants ${ }^{8}$ and mkdifflc (Gal-Yam et al. 2004, 2008). The two reductions gave consistent results. Errors were estimated by first placing artificial sources of the same brightness and at the same distance from the galaxy center as the $\mathrm{SN}$ and then measuring the scatter in measured magnitudes. Finally, the zero point was calibrated with reference magnitudes of stars from the Sloan Digital Sky Survey (Adelman-McCarthy et al. 2007).

We triggered Target of Opportunity observations to obtain spectra with the Double Beam Spectrograph (Oke \& Gunn 1982) on the Hale 200 inch $(5 \mathrm{~m})$ telescope. Two spectra were obtained around maximum light (UT 2007 March 29 and March 30) and a third a fortnight later (April 13). Spectra were taken using the red grating 158/7500, blue grating 300/3990, and a dichroic to split the light at $5500 \AA$. This gave us a total wavelength coverage of 3800-9000 $\AA$ and dispersion of 4.9

\footnotetext{
${ }^{8}$ See http://www.astro.washington.edu/becker/hotpants.html.
} 


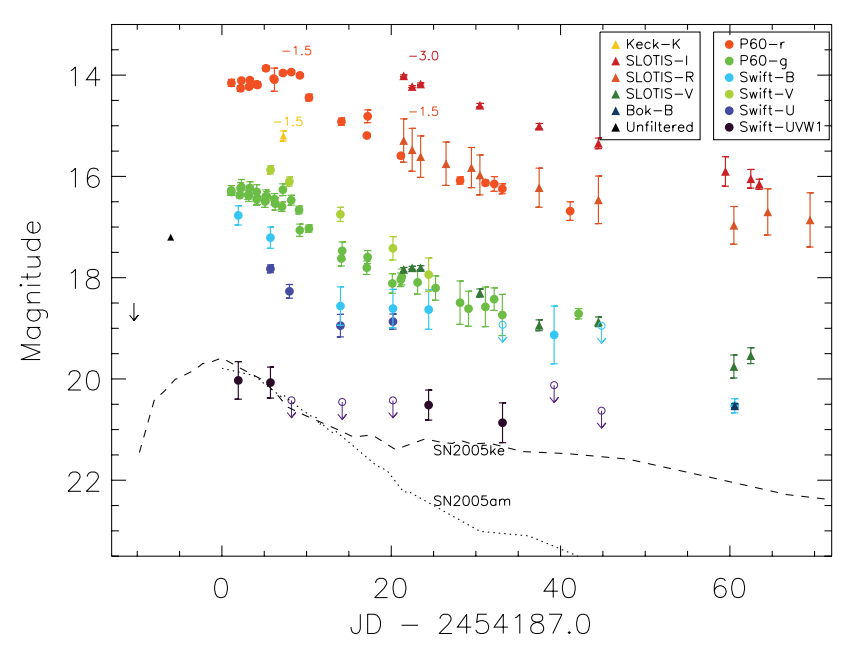

FIG. 1.-Multiband light curve of SN 2007ax based on data from P60, Swift UVOT, SLOTIS, Bok, and Keck II NIRC2. Unfiltered magnitudes from Arbour (2007). Note that similarly to subluminous SN 2005ke (dashed line), SN 2007ax also appears to show an excess in UV emission at $t>20$ days while typical SNe Ia (SN 2005am; dotted line) continue to decline.

and $2.1 \AA$ pixel $^{-1}$ on the red and blue side, respectively. Data were reduced using the standard $\operatorname{IRAF}^{9}$ package apall.

We triggered Swift Target of Opportunity observations for SN 2007ax starting UT 2007 March 29.84 and obtained eight epochs of roughly $5 \mathrm{ks}$ each distributed between the $u v w 2$, $u v m 2, u v w 1, u, b$, and $v$ bands. We also obtained a reference image over 8 months after peak to subtract galaxy light. Aperture photometry was performed using a $3^{\prime \prime}$ circular radius. To estimate the galaxy brightness at this location, a 3 " aperture at the supernova position in the reference image was used. Poole et al. (2008) photometric zero points were applied after appropriately scaling for aperture size. For consistency with calibration, a 5" aperture was used in the computation of coincidence loss. The supernova is detected in $u v w 1$ in four epochs, and not detected in the $u v w 2$ and $u v m 2$ filters. The $b$ band light curve was independently reduced using image subtraction with consistent results. We note that due to the faintness of the supernova and brightness of galaxy background, coincidence loss is dominated by the galaxy light and not a point source, possibly introducing a systematic error in the Swift $u, b$, and $v$ bands.

Further late-time $B V R I$ observations were obtained using the SLOTIS and Bok telescopes and light curves were obtained using image subtraction based on ISIS and IRAF routines. We also obtained near-infrared $K^{\prime}$ imaging using the Keck NIRC2

\footnotetext{
${ }^{9}$ IRAF is distributed by the National Optical Astronomy Observatories, which are operated by the Association of Universities for Research in Astronomy, Inc., under cooperative agreement with the National Science Foundation.
}

instrument with Natural Guide Star adaptive optics on UT 2007 April 4.

\section{ANALYSIS}

We present analysis of the optical and ultraviolet light curve and optical spectrum of SN 2007ax below. We also compare it to other subluminous SN Ia. We adopt a distance modulus of 32.2 (B. Tully 2007, private communication) ${ }^{10}$ to NGC 2577.

\subsection{Optical Light Curve}

We plot the multiband light curve of SN 2007ax in Figure 1. The key characteristic of SN 2007ax is its rapid decline. Traditionally, $\Delta m_{15}$ (the difference between the peak $B$ magnitude and the $B$ magnitude 15 days after the peak) has been used to parameterize the decline of the light curve. However, this parameter can be misleading when applied to the faint $\mathrm{SNe}$ Ia because the knee in their light curve (transition from fast initial decline to slow late-time decline) is sooner than 15 days from the peak. Therefore, we choose to compare the light curves of subluminous Ia using three parameters first introduced by Pskovskii (1984): initial slope $(\beta)$, late-time slope $(\gamma)$, and the time of intersection of the two slopes $\left(t_{b}\right)$. This time of intersection parameter (defined from maximum in $B$ magnitude) was also used by Hamuy et al. (1996) as $t_{2}^{B}$ and shown to be empirically proportional to $\Delta m_{15}$ for some SNe Ia.

For the subclass of faint $\mathrm{SNe}$ Ia, we find that $t_{b}$ is better correlated with the peak absolute $B$ magnitude than the $\beta$ and $\gamma$ slopes of the $B$-band light curve. We fit an empirical relation to the intersection time as a function of peak absolute magnitude and find that $M_{B}=-13.7( \pm 0.5)-0.22( \pm 0.03) \times t_{b}$. Moreover, this transition to slower decline should represent the time at which the optical depth to thermalized radiation becomes thin. We report these three parameters for a sample of subluminous $\mathrm{SNe}$ Ia in Table 1 and show the linear fits in Figure 2.

Another crucial property of subluminous $\mathrm{SNe}$ Ia is that the fainter they are, the redder they are at maximum. We find that SN 2007ax is consistent within uncertainties of the empirical relation derived first by Garnavich et al. (2004): $M_{B}=-18.7$ $+(B-V)_{\max } \times 2.68( \pm 0.32)$. This relation predicts a color in the range of 1.0-1.3 mag and we observe $1.2 \pm 0.1 \mathrm{mag}$. This color has been derived based on synthetic photometry of the spectra around maximum.

\subsection{Ultraviolet Light Curve}

In Figure 1, we compare the Swift UVOT light curve of SN 2007ax to another subluminous SN Ia 2005ke (Immler et al. 2006) and a typical SN Ia 2005am (Brown et al. 2005). The key similarity between SN 2005ke and SN 2007ax is that both

${ }^{10}$ Extragalactic Distance Database, http://edd.ifa.hawaii.edu/.

TABLE 1

CoMParison OF FAINT SNe Ia

\begin{tabular}{|c|c|c|c|c|c|c|c|c|}
\hline Supernova & Galaxy & DM & $\begin{array}{l}M_{B, \max } \\
\text { (mag) }\end{array}$ & $\begin{array}{c}\alpha \\
\left(\text { mag day }^{-1}\right)\end{array}$ & $\begin{array}{c}\beta \\
\left(\text { mag day }^{-1}\right)\end{array}$ & $\begin{array}{c}t_{b} \\
\text { (days) }\end{array}$ & $\begin{array}{l}(B-V)_{\max } \\
\quad(\mathrm{mag})\end{array}$ & Reference \\
\hline SN 2007ax ....... & NGC 2577 & 32.2 & $-15.9 \pm 0.2$ & 0.16 & 0.04 & 10.3 & 1.2 & This Letter \\
\hline SN 1991bg ...... & NGC 4374 & 31.2 & $-16.6 \pm 0.3$ & 0.16 & 0.03 & 14.8 & 0.8 & $\begin{array}{l}\text { Leibundgut et al. (1993), } \\
\text { Filippenko et al. (1992) }\end{array}$ \\
\hline SN 1998de ....... & NGC 252 & 34.3 & $-16.8 \pm 0.2$ & 0.18 & 0.03 & 14.5 & 0.7 & Modjaz et al. (2001) \\
\hline SN $2005 \mathrm{ke} . . . .$. & NGC 1371 & 31.8 & $-17.0 \pm 0.2$ & 0.15 & 0.02 & 14.9 & 0.7 & Immler et al. (2006) \\
\hline SN $2005 \mathrm{bl} \ldots \ldots$ & NGC 4070 & 35.1 & $-17.2 \pm 0.2$ & 0.18 & 0.03 & 14.0 & 0.6 & Taubenberger et al. (2008) \\
\hline SN 1999by ...... & NGC 2841 & 30.9 & $-17.3 \pm 0.2$ & 0.18 & 0.02 & 16.0 & 0.5 & Garnavich et al. (2004) \\
\hline
\end{tabular}




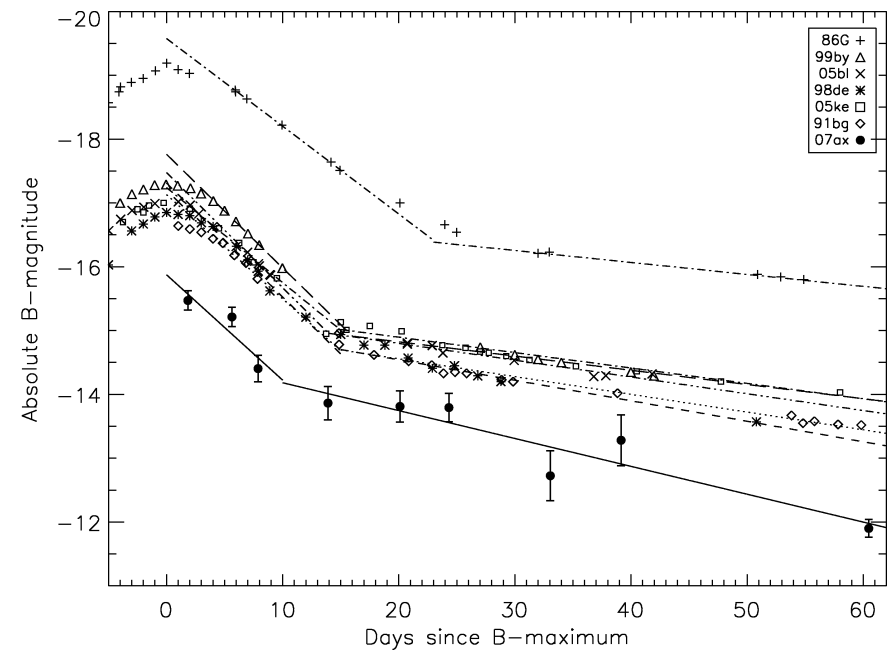

FIG. 2.- $B$-band light curve of SN 2007ax in comparison with other subluminous SNe Ia. The best linear fits are overplotted and give the early-time and late-time slopes. We note that the time of intersection $t_{b}$ of the early-time and late-time slopes is more strongly correlated with the absolute magnitude than the slopes, $\alpha$ and $\beta$.

show an excess in UV starting $\approx 20$ days after the peak. Immler et al. (2006) propose that SN 2005ke showed a UV excess due to circumstellar interaction. Perhaps, subluminous supernovae are optically thin below $3800 \AA$ simply due to lower production of iron-group elements. The question of whether UV excess is a more general property of faint SNe Ia merits further investigation with timely follow-up of a larger sample. With a larger sample, one could also consider whether the break in the UV light curve also depends on absolute magnitude.

\subsection{Spectral Evolution}

We compare optical spectra of SN 2007ax to SN 1991bg in Figure 3. The prominent absorption features are Ti II, O I, Si II, and $\mathrm{Ca}$ I. The presence of intermediate-mass elements like oxygen and titanium is indicative of the presence of unburned material or a low burning efficiency. The absorption features become broader as the supernova evolves. Comparing our spectra to SN 1991bg 1 day, 2 days, and 16 days after maximum in $B$ band, we find that the spectra are very similar. In the first epoch, we see a hint of carbon in the small bump immediately redward of the Si II feature at $6150 \AA$. However, the signalto-noise ratio in the spectrum is too low for any conclusive evidence.

Using the technique described by Nugent et al. (1995) we estimate the temperature diagnostic $R(\mathrm{Si}$ II $)$ - the ratio of the depths of the two Si II features at 5800 and $6150 \AA$ - to be 0.33 . This is smaller than what is implied by the empirical relations derived by Garnavich et al. (2004) and Taubenberger et al. (2008).

We also measure the velocity of the Si II $6150 \AA$ line in the two epochs around maximum and we obtain 9300 and 8800 $\mathrm{km} \mathrm{s}^{-1}$. This is consistent with lower velocities observed in other faint SNe Ia (Benetti et al. 2005).

\subsection{NIR Imaging and Extinction}

We measure a $K^{\prime}$ magnitude of $16.7 \pm 0.1$ on UT 2007 April 4. We determined the contribution of galaxy light at the supernova position by fitting a Sérsic profile to the galaxy using GALFIT (Peng et al. 2002). The best-fit parameters are a Sérsic index of 1.90 , axis ratio of 0.60 , effective radius of $4.98^{\prime \prime}$, position angle of $105.6^{\circ}$, and diskiness of -0.14 . We find no

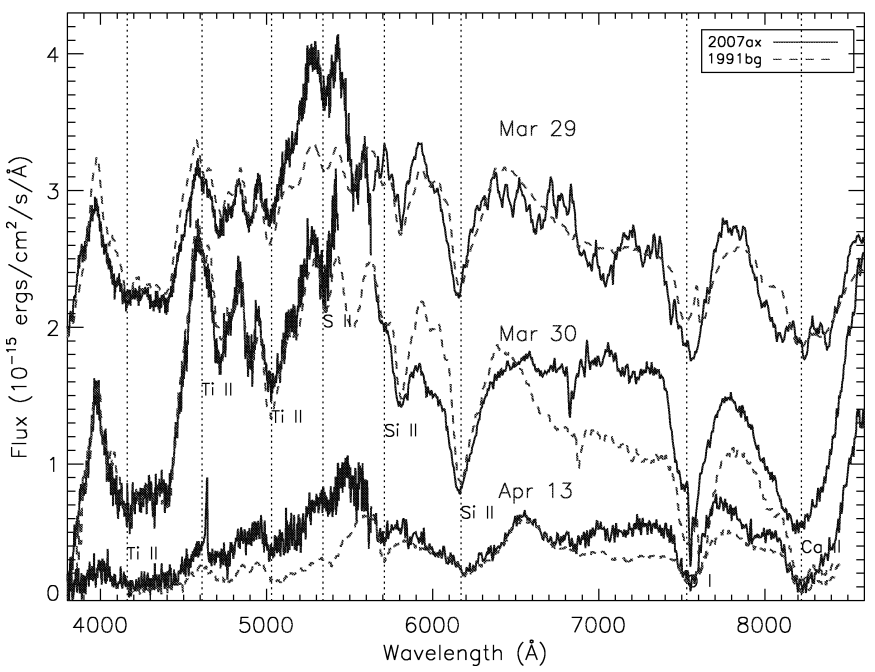

FIG. 3.-Three epochs of P200 DBSP spectra of SN 2007ax (with arbitrary vertical offsets for clarity). Overplotted is another subluminous Type Ia supernova, SN 1991bg, 1 day, 2 days, and 16 days after the peak (scaled by a multiplicative factor for comparison). [See the electronic edition of the Journal for a color version of this figure.]

evidence of dust lanes in this image, suggesting that the host extinction is minimal. This is also consistent with the absence of the interstellar Na D line at $5893 \AA$. We compute an upper limit on the equivalent width as $0.1 \AA$. Using the relations derived in Turatto et al. (2003) we get an upper limit of $E(B-V)<0.01 \mathrm{mag}$ on the extinction.

Based on the Galactic $l=201.1^{\circ}, b=29.6^{\circ}$, the extinction along the line of sight is $E(B-V)=0.054 \mathrm{mag}$ (Schlegel et al. 1998). Therefore, we account for $A_{B}=0.23$ and $A_{v}=$ 0.18 in our calculations of absolute magnitude and luminosities.

\subsection{Bolometric Luminosity and ${ }^{56} \mathrm{Ni}$ Mass}

Arnett et al. (1985) gives an estimate of the ${ }^{56} \mathrm{Ni}$ mass in the ejecta using the peak bolometric luminosity and the rise time:

$$
M_{\mathrm{Ni}}=L_{43}\left[6.31 \exp \left(-t_{r} / 8.8\right)+1.43 \exp \left(-t_{r} / 111\right)\right]^{-1} .
$$

For SN 2007ax, the extinction-corrected peak bolometric luminosity is $2.3 \times 10^{42} \mathrm{ergs} \mathrm{s}^{-1}$. We estimate this by using the photometric points to calibrate our spectrum near maximum light and integrating. The rise time is unknown and unfortunately the literature somewhat arbitrarily assumes 17 days for faint SNe Ia and 19.5 days for typical SNe Ia. Recently, Taubenberger et al. (2008) used SN 1999by early-time data to estimate a rise time of 14 days. The only observational constraint we have for SN 2007ax is that the rise time is longer than 6 days. Thus, for the range of rise times from 6 to 14 days, we find a ${ }^{56} \mathrm{Ni}$ mass of $0.05-0.09 M_{\odot}$. This is consistent with other techniques for estimating ${ }^{56} \mathrm{Ni}$ of faint $\mathrm{SNe} \mathrm{Ia}-$ for SN 1991bg, Cappellaro et al. (1997) model the $V$-band light curve and obtain a mass of $0.1 M_{\odot}$, and Mazzali et al. (1997) model the photospheric and nebular-epoch spectra and obtain a ${ }^{56} \mathrm{Ni}$ mass of $0.07 M_{\odot}$.

\section{DISCUSSION}

To summarize, the primary observational characteristics of subluminous SNe Ia (of which SN 2007ax is an extreme case) are small $t_{b}$ in the optical $B$-band light curve, extremely red $B-V$ color at maximum, possible excess in UV emission at late time, presence of intermediate-mass elements in spectra, 
medium ejecta velocities, low ${ }^{56} \mathrm{Ni}$ mass in ejecta, and short rise times.

Several theoretical models have been proposed to explain faint SNe Ia: complete detonation of a sub-Chandrashekharmass white dwarf, a delayed detonation model, a failed neutron star model, and a small-scale deflagration model. The detonation of a sub-Chandrashekhar C-O white dwarf (e.g., Livne 1990; Woosley \& Weaver 1994) produces more ${ }^{56} \mathrm{Ni}$ than observed and is more blue at maximum than observed (Hoeflich $\&$ Khokhlov 1996). If we consider detonation of a sub-Chandrashekhar O-Ne-Mg white dwarf (Isern et al. 1991), the total nuclear energy is smaller and the predicted ejecta velocities are lower than observed (Filippenko et al. 1992). Mazzali et al. (2007) use detailed spectral modeling to show a common explosion mechanism for all SNe Ia, likely delayed detonation. The failed neutron star model (Nomoto \& Iben 1985) suggests that if the accretion rate of carbon and oxygen from a companion onto a white dwarf is high enough, it may prematurely ignite $\mathrm{CO}$ on the white dwarf surface. Thus, instead of a neutron star, we may see a faint SNe Ia. Small-scale deflagration models suggest that either the burning is restricted to the outer layers or that it occurs slowly.

Another intriguing theoretical possibility recently proposed by Bildsten et al. (2007) is faint thermonuclear supernovae from ultracompact double degenerate AM CVn systems. This supernova is tantalizingly at the brightest end of their predictions $\left(M_{V}=-14\right.$ to -16 , timescale $=2-6$ days, $\left.M_{\mathrm{ej}}<0.1 M_{\odot}\right)$. However, the decay time predicted by these models is much shorter and the ${ }^{56} \mathrm{Ni}$ mass less than that observed in SN 2007ax. Also, the spectrum does not show any feature which suggests being powered by different radioactive material $\left({ }^{48} \mathrm{Cr},{ }^{44} \mathrm{Ti},{ }^{52} \mathrm{Fe}\right)$ produced by some of these models.

None of the above models convincingly explain all the observed characteristics of subluminous SNe Ia. SN 2007ax compels the question of what is the (and whether there is a) lower limit of ${ }^{56} \mathrm{Ni}$ mass in a thermonuclear explosion. Only if we can explain the extremely faint (and the extremely bright) supernovae will we thoroughly understand the limitations in physical processes involved in the thermonuclear explosion, in particular, the ${ }^{56} \mathrm{Ni}$ mass production.

Future supernova surveys which have a shorter cadence and a deeper limiting magnitude will provide invaluable clues to understanding the nature of subluminous SNe Ia. Follow-up of these supernovae with well-sampled UV light curves and well- calibrated multiepoch UV spectra would also be important to understand the apparent excess at late time.

We suggest how a near-future survey, for example, the Palomar Transient Factory, ${ }^{11}$ can systematically search for faint SNe Ia. The parameters of the survey design are sky coverage, cadence, depth, filter, and choice of pointings. Howell (2001) shows that faint $\mathrm{SNe}$ Ia occur preferentially in early-type galaxies and Taubenberger et al. (2008) suggest that they occur in lower metallicity, old stellar mass populations. Since they decline by a magnitude in 5 days, the cadence of the search should be faster than 5 days so that the detection sample is complete. Since faint $\mathrm{SNe}$ Ia are extremely red at maximum, we should choose a red filter for the search. To maximize sky coverage, searching with a single red filter should suffice (with multiband follow-up). Since the local universe is clumpy (e.g., $\approx 25 \%$ of the total light at the distance of Virgo is in the Virgo supercluster), the sky coverage must include concentrations in stellar mass, such as the Virgo, Perseus, and Coma galaxy clusters. The rate of normal SNe Ia is 3 per $10^{11} L_{\odot}$ per century (Scannapieco $\&$ Bildsten 2005). Li et al. (2001) estimate a rate for subluminous SNe Ia to be $16 \%$ of the normal SNe Ia rate based on the LOSS and BAOSS surveys. To a depth of absolute magnitude of -15.5 , and with a limiting magnitude of 20.5 , the survey volume would be $1.5 \times 10^{7} \mathrm{Mpc}^{3}$. Using the 2MASS $K$-band luminosity function of $5.1 \times 10^{8} L_{\odot} \mathrm{Mpc}^{-3}$ (Karachentsev \& Kutkin 2005; Kochanek et al. 2001), we expect a rate of the faintest subluminous supernovae to be $\approx 370$ all sky per year. The Palomar Transient Factory plans a 5 day cadence $2700 \mathrm{deg}^{2}$ experiment which would give $\approx 24$ faint $\mathrm{SNe}$ Ia per year.

We thank Nick Scoville, Milan Bogoslavejic, and the Swift team for performing our Target of Opportunity observations flawlessly. We would like to thank Brent Tully for providing his catalog of nearby galaxies. L. B. acknowledges NSF grants PHY 05-51164 and AST 07-07633. Some of the data presented herein were obtained at the W. M. Keck Observatory, which is operated as a scientific partnership among the California Institute of Technology, the University of California, and the National Aeronautics and Space Administration. The Observatory was made possible by the generous financial support of the W. M. Keck Foundation.

\section{Facilities: PO:1.5m, Hale (DBSP), Keck:I (LRIS), Keck:II (NIRC2), Swift (UVOT), Bok}

\footnotetext{
${ }^{11}$ The Palomar Transient Factory is a dedicated time-domain astronomy project to come online on the Palomar 48 inch in 2008 November.
}

\section{REFERENCES}

Adelman-McCarthy, J. K., et al. 2007, ApJS, 172, 634

Alard, C., \& Lupton, R. H. 1998, ApJ, 503, 325

Arbour, R. 2007, CBET, 904, 1

Arnett, W. D., Branch, D., \& Wheeler, J. C. 1985, Nature, 314, 337

Benetti, S., et al. 2005, ApJ, 623, 1011

Bildsten, L., et al. 2007, ApJ, 662, L95

Blondin, S., et al. 2007, CBET, 907, 1

Brown, P. J., et al. 2005, ApJ, 635, 1192

Cappellaro, E., et al. 1997, A\&A, 328, 203

Cenko, S. B., et al. 2006, PASP, 118, 1396

Filippenko, A. V., et al. 1992, AJ, 104, 1543

Gal-Yam, A., et al. 2004, ApJ, 609, L59 2008, ApJ, 680, 550

Garnavich, P. M., et al. 2004, ApJ, 613, 1120

Hamuy, M., et al. 1996, AJ, 112, 2438

Hoeflich, P., \& Khokhlov, A. 1996, ApJ, 457, 500

Howell, D. A. 2001, ApJ, 554, L193

Immler, S., et al. 2006, ApJ, 648, L119

Isern, J., Canal, R., \& Labay, J. 1991, ApJ, 372, L83

Karachentsev, I. D., \& Kutkin, A. M. 2005, Astron. Lett., 31, 299

Kochanek, C. S., et al. 2001, ApJ, 560, 566
Leibundgut, B., et al. 1993, AJ, 105, 301

Li, W., et al. 2001, ApJ, 546, 734

Livne, E. 1990, ApJ, 354, L53

Mazzali, P. A., et al. 1997, MNRAS, 284, 151

2007, Science, 315, 825

Modjaz, M., et al. 2001, PASP, 113, 308

Morrell, N., \& Folatelli, G. 2007, CBET, 908, 1

Nomoto, K., \& Iben, I., Jr. 1985, ApJ, 297, 531

Nugent, P., et al. 1995, ApJ, 455, L147

Oke, J. B., \& Gunn, J. E. 1982, PASP, 94, 586

Peng, C. Y., Ho, L. C., Impey, C. D., \& Rix, H.-W. 2002, AJ, 124, 266

Poole, T. S., et al. 2008, MNRAS, 383, 627P

Pskovskii, Y. P. 1984, Soviet Astron., 28, 658

Scannapieco, E., \& Bildsten, L. 2005, ApJ, 629, L85

Schlegel, D. J., Finkbeiner, D. P., \& Davis, M. 1998, ApJ, 500, 525

Taubenberger, S., et al. 2008, MNRAS, 385, 75

Turatto, M., Benetti, S., \& Cappellaro, E. 2003, in From Twilight to Highlight: The Physics of Supernovae, ed. W. Hillebrandt \& B. Leibundgut (Berlin: Springer), 200

Woosley, S. E., \& Weaver, T. A. 1994, ApJ, 423, 371 